Discussion Paper No. 06-052

Generationen- oder Parteienkonflikt?

Eine empirische Analyse der deutschen Hochschulausgaben

Ulrich Oberndorfer und Viktor Steiner

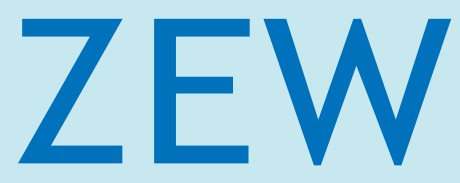

Zentrum für Europäische Wirtschaftsforschung $\mathrm{GmbH}$

Centre for European

Economic Research 
Discussion Paper No. 06-052

\title{
Generationen- oder Parteienkonflikt? Eine empirische Analyse der deutschen Hochschulausgaben
}

\author{
Ulrich Oberndorfer und Viktor Steiner
}

Download this ZEW Discussion Paper from our ftp server:

ftp://ftp.zew.de/pub/zew-docs/dp/dp06052.pdf

Die Discussion Papers dienen einer möglichst schnellen Verbreitung von neueren Forschungsarbeiten des ZEW. Die Beiträge liegen in alleiniger Verantwortung der Autoren und stellen nicht notwendigerweise die Meinung des ZEW dar.

Discussion Papers are intended to make results of ZEW research promptly available to other economists in order to encourage discussion and suggestions for revisions. The authors are solely responsible for the contents which do not necessarily represent the opinion of the ZEW. 


\section{Das Wichtigste in Kürze}

Seit einigen Jahren stehen das deutsche Bildungssystem und damit auch die deutsche Hochschulbildung stark in der öffentlichen Kritik. In der Debatte wird dabei vielfach die Forderung nach einer Verbesserung des staatlichen Bildungsangebots laut. Eine wichtige Komponente können in diesem Kontext die öffentlichen Hochschulausgaben darstellen.

In diesem Beitrag untersuchen wir, ob und in welchem Maße der demographische Wandel sowie unterschiedliche politische Parteienkonstellationen die Hochschulausgaben in den westdeutschen Bundesländern beeinflussen. Einerseits wird vielfach argumentiert, dass zwischen Alterung der Gesellschaft und den Bildungsausgaben ein negativer Zusammenhang besteht. Andererseits unterstellt eine Vielzahl vorwiegend empirischer Untersuchungen, dass linke Parteien höhere Staatsausgaben befürworten als rechte. Ob diese Hypothesen die Entwicklung der Hochschulausgaben in Deutschland erklären können, wurde bisher für Deutschland noch nicht mit Hilfe von ökonometrischen Methoden untersucht. Unsere Analyse leistet dazu einen ersten Beitrag.

Wir finden empirische Evidenz für die Hypothese, dass die Hochschulausgaben negativ von der Bevölkerungsalterung abhängen. Allerdings hängt dieses Ergebnis von der Spezifikation der Demographie-Variablen ab. Unsere Analyse lässt jedoch darauf schließen, dass die Zunahme des Anteils der über 55-jährigen an der Bevölkerung mit einem erheblichen Rückgang der Hochschulausgaben der Länder einhergeht. Der Einfluss unterschiedlicher parteipolitischer Konstellationen auf die Hochschulausgaben kann in diesem Papier sehr klar herausgearbeitet werden.

Im Gegensatz zur These, linke Parteien seien ausgabenfreudiger, lassen unsere Schätzergebnisse jedoch darauf schließen, dass Regierungszeiten mit bürgerlichen Konstellationen sowie der Großen Koalition auf Länderebene mit höheren Hochschulausgaben verbunden sind als jene von SPD-Alleinregierungen. Ein Grund hierfür könnte Klientelpolitik der Parteien sein, wenn man unterstellt, dass etwa bürgerliche Parteien ihre Wählerschaft vornehmlich aus den Schichten rekrutieren, die tendenziell hohe Hochschulausgaben unterstützen. 


\title{
Generationen- oder Parteienkonflikt? Eine empirische Analyse der deutschen Hochschulausgaben
}

\author{
Ulrich Oberndorfer*, Viktor Steiner ${ }^{\dagger}$ \\ *Zentrum für Europäische Wirtschaftsforschung, Mannheim \\ ${ }^{\dagger}$ Deutsches Institut für Wirtschaftsforschung, Berlin, und Freie Universität Berlin
}

Juli 2006

Zusammenfassung: In diesem Beitrag wird auf der Basis von Paneldaten für die westdeutschen Bundesländer untersucht, ob und in welchem Maße der demographische Wandel sowie die unterschiedlichen politischen Parteienkonstellationen die Hochschulausgaben auf Länderebene beeinflussen. Wir finden empirische Evidenz für die Hypothese, dass die Hochschulausgaben negativ von der Bevölkerungsalterung abhängen, allerdings hängt dieses Ergebnis von der Spezifikation der Demographie-Variablen ab. In einer Spezifikation finden wir eine statistisch signifikante Elastizität der Hochschulausgaben bezüglich der Bevölkerungsalterung von größer als -1 . Die geschätzten Effekte unterschiedlicher parteipolitischer Konstellationen auf die Hochschulausgaben sind robust bezüglich alternativer Schätzverfahren sowie Spezifikationen der Demographie-Variablen. Allerdings entsprechen diese nicht der klassischen Partisanentheorie, nach der Parteien des linken Spektrums zu höheren Staatsausgaben neigen. Nach unseren Schätzergebnissen sind Regierungszeiten mit bürgerlichen Konstellationen sowie der Großen Koalition auf Länderebene mit höheren Hochschulausgaben verbunden als jene von SPDAlleinregierungen. Hauptgrund hierfür könnte eine Klientelpolitik der Parteien sein, wenn man unterstellt, dass etwa bürgerliche Parteien ihre Wählerschaft vornehmlich aus den Schichten rekrutieren, die tendenziell hohe Hochschulausgaben unterstützen.

Schlüsselwörter: Demographischer Wandel, Öffentliche Hochschulausgaben, Parteieneffekte

JEL-Klassifikation: H52, H72, I22

Danksagung: Wir danken Dirk Ulbricht und Friedhelm Pfeiffer für hilfreiche Anregungen und Kommentare. Viktor Steiner dankt der Deutschen Forschungsgemeinschaft (DFG) für finanzielle Unterstützung im Rahmen des Forschungsprojekts „Politische Ökonomie dezentraler Bildungsfinanzierung - Theoretische Analyse und empirische Anwendung für Deutschland“" (STE 681/6-1). 


\section{Einleitung}

Seit einigen Jahren steht das deutsche Bildungssystem stark in der öffentlichen Kritik. Grund hierfür sind nicht zuletzt mittelmäßige Ergebnisse in internationalen Vergleichsstudien. Betroffen sind davon auch die deutschen Universitäten, die in länderübergreifenden Hochschulrankings nur selten Eingang in die Spitzengruppen finden. In der Debatte wird vielfach die Forderung nach einer Verbesserung des staatlichen Bildungsangebots laut. Der Bund hat inzwischen darauf reagiert und will mit Hilfe der „Exzellenzinitiative“ so genannte „Leuchttürme“ der höheren Bildung und Forschung in Deutschland stärken. Einige Bundesländer konterkarieren diese Bemühungen jedoch ihrerseits durch Einsparungen bei der Hochschulförderung. Darüber hinaus lehnen die Bundesländer weitgehend unabhängig von den politischen Konstellationen in Bund und Ländern eine stärkere gestalterische und finanzielle Beteiligung des Bundes im Hochschulbereich in seltener Eintracht ab.

Aus ökonomischer Sicht erscheint diese Situation ineffizient (vgl. z.B. Haupt und Janeba, 2003, Steiner, 2002). Obwohl Bildung von allen politischen Parteien als entscheidender Faktor für die zukünftige Entwicklung und internationale Wettbewerbsfähigkeit Deutschlands angesehen wird und Bildungsreformen allgemein als dringlich empfunden werden, weichen die damit verbundenen Vorstellungen zu stark voneinander ab, als dass der Reformstau überwunden werden könnte. Dies gilt für alle Bildungsstufen, insbesondere aber auch im Bereich der Hochschulen, der in Deutschland in die Zuständigkeit der Länder fällt. Eine wichtige Entscheidung betrifft dabei die öffentlichen Ausgaben für die Hochschulen, die überwiegend von den Ländern getragen werden. Da die Entscheidung politischer Mehrheiten in den einzelnen Ländern bedarf, stellt sich die Frage nach den polit-ökonomischen Determinanten der Hochschulausgaben auf Länderebene.

Die mit der staatlichen Bildungsfinanzierung verbundene Umverteilung findet nicht nur zwischen Einkommensklassen, sondern auch - und vor allem - zwischen Generationen statt (Kemnitz und von Weizsäcker, 2003). Es ist zu erwarten, dass dieser intergenerationelle Verteilungskonflikt bei zunehmender Alterung der Bevölkerung aufgrund polit-ökonomischer Entscheidungen mit einem Rückgang der öffentlichen Bildungsausgaben verbunden sein wird. Allerdings ist dies keine zwangsläufige Entwicklung, da der intergenerationelle Verteilungskonflikt zum einen durch gegenläufige Effekte abgeschwächt werden kann. Andererseits wird über staatliche Bildungsausgaben möglicherweise nicht entsprechend den Präferenzen des Medianwählers, sondern nach den Präferenzen bzw. Ideologien unterschiedlicher Parteien entschieden, die entsprechend der so genannten Partisanentheorie (Hibbs, 1977) nicht durch die Macht des Medianwählers eliminiert werden. 
Bisher liegt unseres Wissens für Deutschland keine empirische Studie zum Einfluss politischer Parteienkonstellationen auf die Hochschulausgaben vor. Die vorliegende Untersuchung soll auch klären, ob der demographische Wandel die deutschen Hochschulausgaben beeinflusst, die als Transfer zu Gunsten der Jüngeren interpretiert werden können. Die Effekte der Alterung der Gesellschaft sind in jüngerer Vergangenheit verstärkt in den Fokus wirtschaftswissenschaftlicher Analysen geraten. Deutschland als eines der Industrieländer mit der am stärksten gealterten Bevölkerung steht unter besonderer Beobachtung. Sinn und Uebelmesser (2003) etwa warnen davor, dass notwendige Reformen in baldiger Zukunft aufgrund des Einflusses der Älteren auf politische Entscheidungen nicht mehr durchsetzbar sein werden.

Im nächsten Abschnitt werden zunächst mögliche Effekte der Alterung der Bevölkerung auf die Hochschulausgaben und der Einfluss von Parteien auf die Höhe der Hochschulausgaben herausgearbeitet sowie bisherige empirische Ergebnisse vorgestellt. Anschließend stellen wir das föderalstaatliche Umfeld der Hochschulausgaben in Deutschland, die Datenbasis und den Schätzansatz dar und präsentieren unsere empirischen Ergebnisse. Die Arbeit schließt mit einer Diskussion der Ergebnisse und wirtschaftspolitischen Implikationen.

\section{Politische Ökonomie der Hochschulfinanzierung}

\subsection{Medianwählertheorie und Effekte der Bevölkerungsalterung}

Der Standardansatz für die positive Theorie der öffentlichen Bereitstellung eines Gutes ist das Medianwählertheorem nach Downs (1957). Demnach stellt die Regierung genau die Menge eines Gutes mit Steuermitteln bereit, die der „,mittlere“ Wähler wünscht. Dieser wird in der Regel über das Einkommen definiert. Überträgt man den Ansatz auf die Bildungs- bzw. Hochschulausgaben, so würde der Staat bzw. ein Bundesland genau soviel Steuermittel in den Bereich investieren, wie dies der Medianwähler wünscht. Das Medianwählertheorem gilt allerdings nur unter der Grundannahme der eingipfligen Präferenzen. Diese wäre gegeben, wenn die gewünschte Menge an öffentlich bereitgestellter Bildung mit dem Einkommen der Bürger monoton steigt. Damit die Annahme erfüllt ist, muss sichergestellt sein, dass der Einkommenseffekt den Steuerpreiseffekt dominiert (vgl. Kemnitz und von Weizsäcker, 2003, Borck, 2006). Voraussetzung dafür ist, dass reiche Haushalte höhere Bildungsausgaben als ärmere bevorzugen, obwohl sie damit einen höheren Relativpreis in Kauf nehmen. Nur in einem System, in dem keine privaten Bildungseinrichtungen als Alternativen zum öffentlichen Angebot existieren, gilt dies als plausibel (vgl. z. B. Epple und Romano, 1996). 
Es ist fraglich, ob das Medianwählertheorem auf den deutschen Hochschulbereich angewendet werden kann, da hier durchaus private Alternativen bestehen (Studium im Ausland, zunehmend auch private Hochschulen im Inland).

Das einfache Medianwählermodell zur Erklärung staatlicher Bildungsausgaben vernachlässigt auch das Alter als eine wichtige Determinante der Zustimmung zu den Hochschulausgaben. Dabei findet die durch die Hochschulausgaben induzierte Umverteilung nicht nur zwischen Einkommensklassen statt, sondern auch - und vor allem - zwischen Generationen. Bildungsrelevant sind in der Regel Teile der jungen Generation, die Bildung konsumieren. Sie gehen häufig keiner beruflichen Tätigkeit nach und müssen damit zudem in der Regel nur eine geringe Steuerlast schultern. Die Jungen erhalten damit anhand von Bildung einen Realtransfer, der vor allem durch Ältere über das Steuersystem finanziert wird. Zudem konkurriert das Bildungswesen in öffentlichen Haushalten mit Bereichen wie etwa dem Gesundheitswesen, von denen in besonderem Maße Ältere direkt profitieren. Dies stellt einen weiteren Grund dafür dar, dass Jüngere höhere Bildungsausgaben präferieren könnten als Ältere.

Die These, dass sich Ältere gegen öffentliche Ausgaben für Bildung oder spezieller für Hochschulen aussprechen, ist allerdings nicht unwidersprochen. Eine Zusammenfassung der Gegenargumente liefert Poterba (1998). Als einen wichtigen Grund führt er - neben Altruismus - intergenerationelle Externalitäten an. Bildungsinvestitionen können demnach den gesamtgesellschaftlichen Ressourcenpool erhöhen. Dies kann sich wiederum positiv auf den Nutzen Älterer niederschlagen. Der Nutzenzuwachs erfolgt dadurch, dass besser ausgebildete Personen in der Regel höhere Einkommen erwirtschaften, was höhere Einnahmen der Renten- und/oder Sozialversicherung bedingt. Hinzu kommt das Argument, nach dem die Bildungsausgaben die Qualität der Dienstleistungen steigern, die Jüngere den Älteren anbieten. Dies wird etwa am Beispiel der Ausbildung von Medizinern und Altenpflegern besonders deutlich. Die Argumentation wird allerdings dadurch eingeschränkt, dass die Jüngeren selbst vom oben genannten Ressourcenpool profitieren können, und das sogar in stärkerem Maße als die Älteren. Mit steigendem Alter verkürzt sich der Zeitraum, in dem ein Individuum von staatlicher Bildungsförderung profitieren kann. Nach Kemnitz (2000) sorgt dies für einen negativen Zusammenhang zwischen Alter und der gewünschten Höhe des Bildungsetats.

Auch Gradstein und Kaganovich (2004) greifen die These an, dass der demographische Wandel zu einem starken Rückgang der Bildungsausgaben über die Zeit führt. Dies schreiben sie einem gegenläufigen polit-ökonomischen Effekt zu. Demnach antizipieren die Jüngeren finanzielle Defizite in der Rentenkasse, die aus mangelnder Bildung noch jüngerer 
Generationen herrühren. Für sie besteht daher ein Anreiz, in gealterten Gesellschaften für höhere Bildungsausgaben $\mathrm{zu}$ stimmen als in jungen. Dieser Effekt wird noch dadurch verstärkt, dass die Jungen ihre steigende Lebenserwartung mit ins Kalkül einbeziehen.

Die angeführten Gegenargumente scheinen die Theorie der intergenerationellen Differenzen bezüglich der Verteilung öffentlicher Mittel jedoch höchstens abschwächen, nicht aber widerlegen zu können. Spätestens ab einem gewissen Altersniveau ist mit einer abnehmenden Präferenz für öffentlich finanzierte Hochschulbildung zu rechnen. Bleibt die Einkommensverteilung in einer Gesellschaft konstant und damit der Disput bezüglich Einkommen und präferierten Bildungsausgaben unberücksichtigt, während der Anteil Älterer an der Bevölkerung zunimmt, so wird der pivotale Wähler hinsichtlich der Hochschulausgaben demnach verschoben. Dies impliziert geringere Hochschulausgaben. Tritt der Fall ein, dass der demographische Wandel nicht über die beschriebene Wirkungskette den pivotalen Wähler verschiebt, so kann er dennoch für eine Absenkung des bereitgestellten Niveaus an Hochschulbildung führen. Kemnitz (2000) zeigt theoretisch auf, dass die Alterung von Gesellschaften höhere Ungleichheit verursacht. Aus diesem Zusammenhang kann wiederum ein ärmerer Medianwähler resultieren. Selbst wenn der demographische Wandel also den pivotalen Wähler nicht direkt verschiebt oder gar das Medianwählertheorem auf die (höhere) Bildung anwendbar ist, weil ein monotoner Zusammenhang zwischen Einkommen und präferiertem Niveau an Hochschulbildung existiert, kann nach der positiven Theorie ein wachsender Anteil Älterer an der Bevölkerung dazu führen, dass die Bildungsausgaben sinken.

Poterba (1997) identifiziert moderate Anzeichen eines intergenerationellen Konflikts bezüglich der Schulausgaben in den Vereinigten Staaten. Er zeigt, dass eine Zunahme des Anteils älterer Personen (über 65 Jahre) an der Bevölkerung auf der Ebene der Bundesstaaten mit einer signifikanten Reduzierung der Schulausgaben (pro Kind im Alter von fünf bis 17 Jahren) einhergeht. Dabei berücksichtigt er sowohl die Ausgaben auf Bundesstaaten- als auch auf lokaler Ebene. Die von ihm geschätzte Elastizität beträgt -0,25. Ähnliche Ergebnisse bezüglich des Einflusses Älterer auf die Schulausgaben auf State-Ebene berichten auch Fernandez und Rogerson (2001), während Ladd und Murray (2001) zwar einen negativen Zusammenhang zwischen Anteil der Älteren an der Bevölkerung und Schulausgaben auf der Ebene der Bundesstaaten, nicht aber auf Bezirksebene (Counties) finden. Harris et al. (2001) ermitteln zwar auf der Ebene der Bezirke einen negativen Zusammenhang zwischen Alterung der Bevölkerung und den Schulausgaben pro Schüler; dieser fällt aber deutlich schwächer aus als der von Poterba geschätzte. 
Für Deutschland haben Baum und Seitz (2003) die Pro-Kopf-Bildungsausgaben für westdeutsche Flächenländer auf Gemeindeebene analysiert. Die Arbeit ist die bisher einzige Studie, die den Zusammenhang zwischen demographischem Wandel und Bildungsausgaben für Deutschland empirisch untersucht. Die Autoren erhalten nur geringe empirische Evidenz für intergenerationelle Konflikte bezüglich dieser öffentlichen Ausgaben. Bei den gesamten Schulausgaben ist der geschätzte Koeffizient für die „Altenquote“ nur in den Gleichungen signifikant negativ, die - auf Gemeindeebene konsolidiert - sowohl Ausgaben der Gemeinden als auch der Länder oder aber nur die Länderausgaben erklären. Die Spezifikation, in der die Autoren demographische Kennziffern aus einzelnen Gemeinden verwenden, um die Ausgaben der Länder für Schulen in diesen Gemeinden zu erklären, ist institutionell gesehen nicht vollständig kohärent. Für Baum und Seitz' Analyse ergeben sich daher zumindest für die konsolidierte Schätzung ähnliche Probleme wie für Poterba (1997).

\subsection{Parteien- und Ideologieeffekte}

Im Grundmodell der Medianwählertheorie spielen Präferenzen bzw. Ideologien von unterschiedlichen Parteien keine Rolle. Demnach müssen alle zur Wahl stehenden Parteien die Präferenzen des Medianwählers in ihrem Wahlprogramm berücksichtigen, um überhaupt die Chance zu besitzen, die Abstimmung für sich $\mathrm{zu}$ entscheiden. In einigen Modellerweiterungen kommen jedoch Parteipräferenzen bzw. Ideologien ins Spiel. Diese lassen sich nicht durch die Macht des Medianwählers eliminieren, wenn die ins Amt gewählten Kandidaten nicht zwingend ihr Wahlprogramm in tatsächliche Politik umsetzen müssen (vgl. Alesina, 1988). Die Grundlage für den parties-do-matter-Ansatz, auch Partisanentheorie genannt, legt Hibbs (1977). Er argumentiert, dass rechte Parteien höhere Einkommensschichten repräsentierten, die vor allem an geringen Inflationsraten, weniger aber an niedrigen Arbeitslosenquoten interessiert seien, und sich hingegen linke Parteien eher für Geringverdiener und sozial Schwache einsetzen. Demnach favorisieren linke Parteien höhere Ausgaben als rechte bzw. konservative Parteien. Die Partisanentheorie ist analog auf Teilbereiche der Staatsausgaben wie die Hochschulausgaben anwendbar.

Empirische Evidenz für Partisaneneffekte auf die Staatsausgaben insgesamt finden z.B. für die OECD-Länder Roubini und Sachs (1989), Blais, Blake und Dion (1993) und Cusack (1997). Nach Cusack lassen sich mehr als 40 Prozent deren Variation im Querschnitt von 1970 lassen sich durch Unterschiede in der Regierungszusammensetzung erklären. Die völlige Regierungskontrolle durch die Linke erhöht dabei den Bildungsanteil am BIP um elf Prozent. Für das folgende Jahrzehnt liefert die Untersuchung ähnliche Werte, die sich jedoch 
statistisch nicht signifikant von Null unterscheiden. Bezogen auf staatliche Bildungsausgaben findet Boix (1997) ebenfalls für die OECD-Länder Parteieneffekte.

Für Westdeutschland hat Seitz (2000) den Zusammenhang zwischen der Höhe der Länderdefizite und die parteipolitische Zusammensetzung der jeweiligen Regierung untersucht. Die Analyse der Defizite begründet er mit der Tatsache, dass die deutschen Länder aus institutionellen Gründen keine Steuersätze als frei wählbares Politikinstrument besitzen. Dadurch müssten sie sich der Ausgaben und damit Defizite als Politikvariablen bedienen. Diese Arbeit liefert jedoch keine Evidenz dafür, dass politische Ideologien makroökonomische Tendenzen erklären. Seitz kann keinen signifikanten Einfluss von Parteien bzw. Koalitionen auf die Fiskalpolitik feststellen.

$\mathrm{Zu}$ einem ähnlichen Ergebnis hinsichtlich des Zusammenhangs zwischen Länderdefiziten und politischen Konstellationen in Deutschland kommen auch Jochimsen und Nuscheler (2004). Ihre Ergebnisse unterscheiden sich diesbezüglich jedoch beim Test auf politischen Opportunismus. Die Autoren finden, dass das Defizitwachstum im jeweiligen Jahr vor Landtagswahlen zurückgeht. Erwartungsgemäß ist dieser Effekt unter CDUAlleinregierungen, noch stärker aber bei sozial-liberalen Koalitionen messbar. Generelle Belege für die Partisanentheorie erhalten die Autoren damit nicht. Dennoch lassen die Ergebnisse darauf schließen, dass es parteipolitische Unterschiede bezüglich der durchgesetzten Fiskalpolitik gibt - wenn auch nur in den Jahren, die den Wahlen direkt vorausgehen.

Rodden (2001) ist der einzige Autor, der allgemeine Partisan-Effekte für Deutschland ermitteln kann. Bei seiner Untersuchung der westdeutschen Bundesländer ohne Berlin im Zeitraum von 1974 bis 1995 kommt er zu dem Schluss, dass konservative Länder übereinstimmend mit der Theorie - weniger ausgeben und geringere Defizite verursachen als linksorientierte Regierungen. Weiterhin ergeben Roddens Schätzungen, dass die Länder in Wahljahren höhere Ausgaben und Defizite verantworten als in sonstigen Jahren. Unterschiede zwischen den Parteien kann der Autor hierbei im Gegensatz zu Jochimsen und Nuscheler (2004) aber nicht feststellen.

Zum aktuellen Zeitpunkt gibt es noch keine Studie, die den Zusammenhang zwischen politischen Konstellationen und den Bildungs- bzw. Hochschulausgaben innerhalb Deutschlands ausführlich untersucht. Schmidt (2003) führt lediglich eine knappe statistische Analyse der mit dem BIP skalierten zusammengefassten Bildungs-, Wissenschafts- und Kulturausgaben für das Jahr 2000 durch. Er aggregiert dafür die Ausgaben der Länder sowie deren untergeordneter Gebietskörperschaften auf Länderniveau. Anstelle von Parteieneffekten 
findet der Autor vor allem große Unterschiede in den Bildungsausgaben zwischen Ost(inklusive Berlin) und Westdeutschland.

\section{Empirische Analyse für Deutschland}

\subsection{Fiskalischer Föderalismus und untersuchte Bundesländer}

In Deutschland sind die Länder für die Hochschulbildung verantwortlich. Knapp 90 Prozent der Gesamtausgaben für die Hochschulen entfallen auf die Länder. Der Bund trägt lediglich die Kosten für den Hochschulbau, was allerdings durch die aktuelle Diskussion um die Föderalismusreform für die Zukunft in Frage gestellt ist. Von den Gemeinden erhalten die Hochschulen keinerlei Zuschüsse (vgl. Seitz, Freigang und Kempkes, 2005).

Die Haupteinnahmen der Länder sind Steuereinnahmen. Den Großteil davon machen wiederum die gemeinsamen Steuern mit dem Bund aus. Die Länder erhalten hierbei Teile der Einkommensteuer und der Mehrwertsteuer. Die Verteilung der ersteren unter den Ländern hängt von dem Aufkommen in dem jeweiligen Land ab, die Mehrwertsteuer wird auf Basis der Bevölkerungsanteile den Staaten zugeteilt. Zudem erhalten die Länder einige Steuereinnahmen, die in ihren Grenzen anfallen, zu 100 Prozent. Dies sind Kraftfahrzeug-, Grunderwerb-, Erbschaft- und einige eher unbedeutende Steuern. Der Bund erhält die gesamten Einnahmen aus der Mineralöl-, Versicherung- und Tabaksteuer sowie weitere unbedeutende Steuern. Städte und Gemeinden erhalten unter anderem die Einnahmen aus Gewerbe- und Grundsteuer.

Eine Besonderheit des deutschen Steuersystems ist die gegenseitige Abhängigkeit von Bund und Ländern bei der Steuersetzung. Die Länder selbst besitzen nicht die Kompetenzen, Steuern zu erheben. Die Sätze werden vom Bund bestimmt, wobei die Änderung eines Satzes, der die Länder betrifft, eines Gesetzes bedarf, dem auch die Länder über den Bundesrat zustimmen müssen. Über diesen Weg können die Länder die Steuerpolitik beeinflussen, aber keines der Länder kann individuell Steuern senken oder erhöhen. Aus der oben geschilderten ersten Aufteilung der gemeinsamen Steuern zwischen Bund und Ländern resultieren erhebliche Einnahmedifferenzen zwischen den Ländern. Um diese abzumildern sind im deutschen System Ausgleichsmechanismen vorgesehen.

Im horizontalen Finanzausgleich (Länderfinanzausgleich) werden Finanzkraft und Finanzbedarf der Länder untereinander angeglichen - Länder, deren Finanzkraft den Bedarf übersteigt, müssen Gelder an die Staaten abgeben, bei denen das Gegenteil der Fall ist. Das Konzept des Bedarfs orientiert sich dabei an den Pro-Kopf-Steuereinnahmen eines Landes. In einer weiteren Stufe unterstützt der Bund die finanzschwächeren Länder über Fehlbetrags- 
Ergänzungszuweisungen. Der Länderfinanzausgleich führt zur Nivellierung der Steuereinnahmen zwischen den Bundesländern mit dem „Ziel eines bundesweit gleichen ProKopf-Angebots öffentlicher Güter“ (Homburg, 1994, S. 314).

In die folgende empirische Analyse werden nur die westdeutschen Länder (ohne Berlin) einbezogen. Strukturelle Unterschiede zwischen den Flächenstaaten und den Stadtstaaten Bremen und Hamburg (vgl. Seitz, 2000) werden in den Panelschätzungen, soweit erforderlich, durch eine Dummyvariable erfasst. Die ostdeutschen Bundesländer können hier nicht berücksichtigt werden, da für diese erst ab 1992 Daten verfügbar sind und die ersten Jahre nach der deutschen Vereinigung von großen Infrastruktur-Investitionen im Hochschulbereich geprägt war. Weiterhin wird auch Berlin aus der Analyse ausgeschlossen. Dies liegt im stark ausgeprägten Strukturbruch in den Jahren 1991/92 begründet, als der Stadtstaat um den ehemaligen Ostteil erweitert wurde. Zudem hatte das damalige West-Berlin nicht am System des Länderfinanzausgleichs teilgenommen und wurde direkt vom Bund finanziert.

\subsection{Daten und Variablen}

\section{Hochschulausgaben}

In dieser Arbeit werden die realen Hochschulausgaben der Länder (Grundmittel) zwischen 1985 und 2002, skaliert mit der jeweiligen Einwohnerzahl (HSK) und deflationiert mit dem deutschen BIP-Deflator in Preisen des Jahres 1996 untersucht. Die GrundmittelHochschulausgaben sind eine sehr heterogene Größe. Sie umfassen die Ausgaben für Lehre und Forschung der Hochschulen ebenso wie deren Ausgaben für Weiterbildung und nichtwissenschaftliche Ausbildung. Zudem werden die Ausgaben der Hochschulkliniken bereinigt um die Krankenbehandlung als Lehr- oder FuE-Aufwendungen einbezogen (vgl. Hetmeier, 2000) ${ }^{1}$.

Im Gegensatz zu einigen Studien über die Schulausgaben von Gebietskörperschaften werden die Hochschulausgaben in dieser Arbeit nicht skaliert mit der Anzahl der Empfänger, also Studierenden, sondern auf Basis der Landesbevölkerung untersucht. Generelle Aussagen anhand der Ausgabenhöhe pro Student sind höchst problematisch. Zum einen schwankt diese Größe über die Hochschultypen - Universitäten, Kunsthochschulen, medizinische Einrichtungen der Universitäten und verschiedene Fachhochschularten - stark. Zum anderen differieren die Ausgaben pro Student auch innerhalb eines Hochschultyps zwischen den Fächern. Hinzu kommt, dass sich in den vergangenen Jahrzehnten die Hochschulstruktur

\footnotetext{
${ }^{1}$ Detaillierte Erläuterungen zur Konstruktion der Variablen finden sich im Anhang A.
} 
Deutschlands in diesem Sinne verändert hat. Die Bedeutung der weniger ausgabeintensiven Fachhochschulen nahm zu und die Fächerstruktur an den Hochschulen variierte laufend (vgl. Hetmeier und Weiß, 2001). Während im Jahr 2001 Studierende an Kunsthochschulen mit Grundmitteln von 12.400 Euro pro Student gefördert wurden, waren Fachhochschulstudenten mit einem Zuschussbedarf von 4.300 Euro vergleichsweise „günstig“. Dazwischen lagen Universitätsstudenten (ohne medizinische Einrichtungen) mit 6.500 Euro. Der Mittelwert betrug 7.200 Euro. Bei einem Vergleich bezüglich der Fächergruppen lagen die Mediziner mit 28.400 Euro pro Student vorne, während Rechts-, Wirtschafts- und Sozialwissenschaftler am Ende der Skala nur mit je 2.070 Euro bezuschusst wurden (vgl. Statistisches Bundesamt, 2003).

Daher wird ein Bundesland, das zwar eine überdurchschnittlich hohe Anzahl von Studenten ausbildet, diesen aber überwiegend kostengünstige Studienplätze anbietet, in einer Pro-Student-Betrachtung geringere Werte aufweisen als ein Bundesland, das relativ wenige „teure“ Studienplätze anbietet. Unterschiedliche oder sich ändernde Strategien von Ländern würden eine Untersuchung der Hochschulausgaben verzerren oder unmöglich machen. Daher scheint eine Pro-Einwohner-Betrachtung geeigneter, Unterschiede in den Hochschulausgaben zu identifizieren. Dem liegt die Annahme zugrunde, jedes Land könne unter identischen Bedingungen gemessen an seiner Einwohnerzahl den gleichen Betrag in seine Hochschulen investieren.

Zusätzlich zu diesen Aspekten spricht gegen eine Betrachtung der Ausgaben pro Studierendem, dass der Schwerpunkt dieser Arbeit auf einer anderen Fragestellung beruht als dies bei den in der Literaturübersicht aufgeführten Untersuchungen der Schulausgaben der Fall ist. Diese analysieren, ob ein Rückgang der Schülerzahlen einen Rückgang an Schulausgaben mit sich bringt. Im Gegensatz $\mathrm{zu}$ den Schülerzahlen ist bei den Studierendenzahlen im Beobachtungszeitraum in Deutschland jedoch kein Rückgang, sondern eine teilweise deutliche Steigerung zu beobachten (vgl. Statistisches Bundesamt, 2003).

Abbildung 1 zeigt, dass die Pro-Kopf-Ausgaben für die Hochschulen unter den Bundesländern erheblich schwanken. Während der Mittelwert des gesamten Samples bei 183 Euro pro Kopf liegt (Standardabweichung: 53,21), gab Rheinland-Pfalz durchschnittlich nur 120 Euro aus, während Hamburg 295 Euro aufwendete (vgl. Anhang C). Es fällt auf, dass die Stadtstaaten bei - vor allem für Bremen - hohen Standardabweichungen deutlich höhere Ausgaben als die Flächenstaaten verbuchten. Über die Zeit stieg in den meisten Ländern das Niveau der Hochschulausgaben pro Kopf bei zunehmender Varianz. Bayern, Rheinland-Pfalz sowie Schleswig-Holstein erlebten jedoch zwischen 1995 und 2002 eine Abnahme der ProKopf-Ausgaben. 


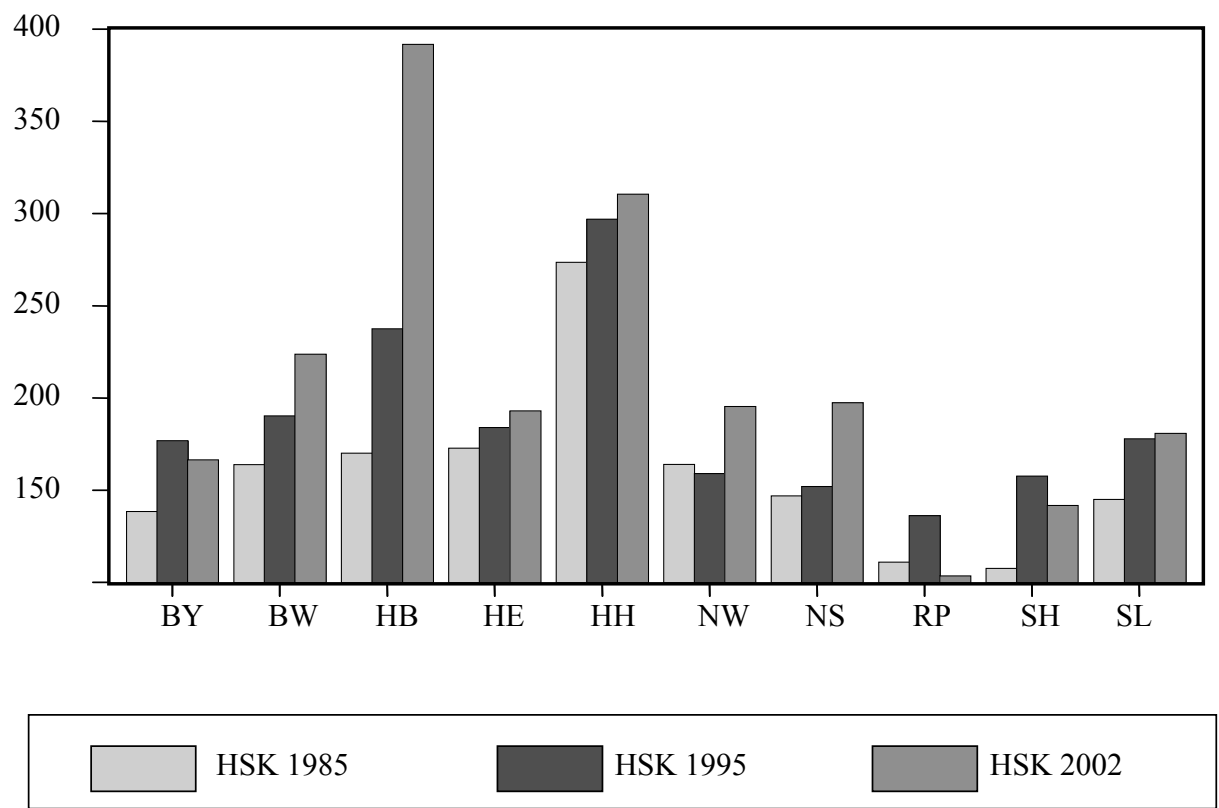

Quelle: Statistisches Bundesamt

\section{Demographie}

Zur Abbildung des demographischen Wandels verwenden wir zwei unterschiedliche Definitionen der Bevölkerungsalterung. Die erste Definition folgt der bestehenden Literatur, die den Anteil der über 65-jährigen verwendet (vgl. Abbildung 2a). Diese Definition ist gut geeignet, da über 65-jährige in der Regel kaum Kinder haben, die noch das staatliche Bildungsangebot in Form von Hochschulen wahrnehmen. Damit wird das in Abschnitt 2 erwähnte Argument des Altruismus zumindest teilweise entkräftet, das besonders stark ausfallen dürfte, wenn „direkte“ Familienangehörige von einer Entscheidung betroffen sind. Dennoch ist die Grenze von 65 Jahren grundsätzlich willkürlich gezogen. Daher wird in dieser Arbeit auch eine alternative Definition der Älteren verwendet, welche die über 55jährigen einbezieht (vgl. Abbildung 2b). Dies lässt sich zum einen mit ähnlichen Argumenten wie bei den über 65-jährigen begründen. Zum anderen verfügt diese Gruppe aufgrund ihres wesentlich größeren Anteils in der Bevölkerung über größeren politischen Einfluss. 
a) Anteil der über 65-jährigen

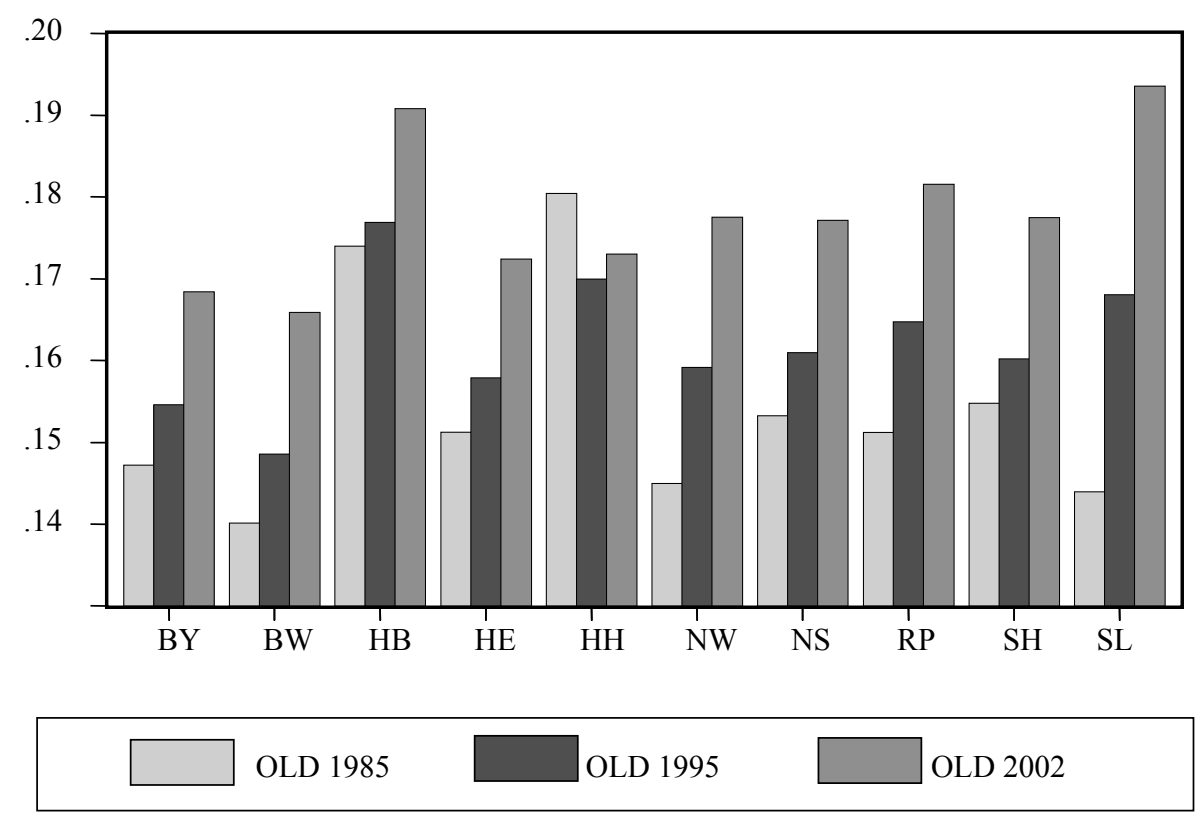

b) Anteil der über 55-jährigen

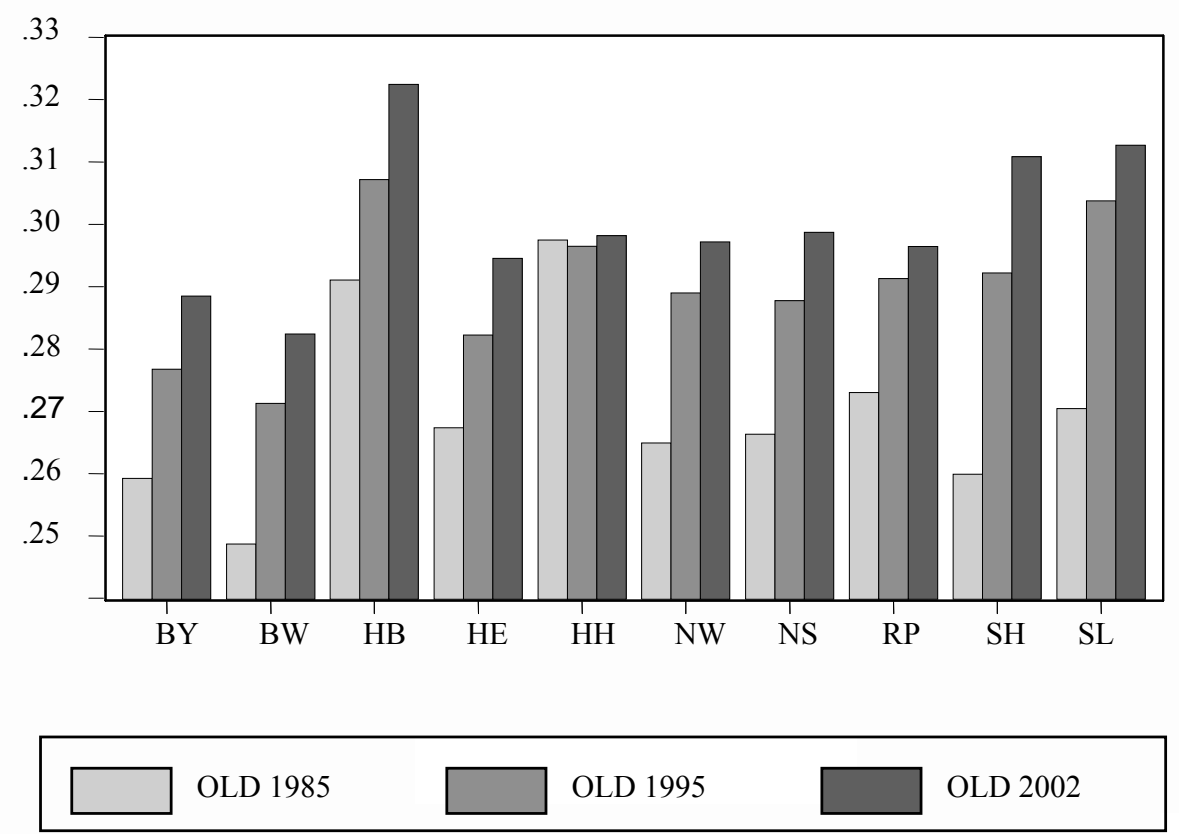

Quelle: Statistisches Bundesamt 


\section{Politikvariablen $^{2}$}

Im Zeitraum zwischen 1985 und 2002 bestimmten sehr unterschiedliche politische Konstellationen die Politik der betrachteten Bundesländer. Die in dieser Arbeit berücksichtigten Alleinregierungen und Koalitionen sind in Tabelle 1 aufgeführt. Es ist anzumerken, dass weitere Koalitionen auf Landesebene bestanden, die nur ein Mal während der Beobachtungsperiode auftraten und/oder mit Parteien gebildet wurden, die nur regionale und keine nationale Bedeutung haben. Diese Fälle werden jeweils spezifisch behandelt und erläutert.

Die in Tabelle 1 dargestellten Politikkonstellationen werden durch Dummy-Variablen erfasst. Diese nehmen für eine bestimmte Koalition (Partei) den Wert „1“ an, wenn diese (alleine) in einem bestimmten Zeitraum die Regierung stellt, und den Wert „0“ sonst. SPDAlleinregierungen stellen dabei die Referenzkategorie dar. Der Wert „1“ wird im Falle eines Regierungswechsels an die Konstellation vergeben, die über den längeren Zeitraum im Beobachtungsjahr an der Macht war. Löste in einem Bundesland beispielsweise im Juli die SPD die CDU als Regierungspartei ab, wird dieses Jahr noch für die CDU ,gezählt“. Anders verhält es sich, wenn der Regierungswechsel bis zum Juni vollzogen wurde. Weiterhin ist zu beachten, dass in dieser Untersuchung nicht zwischen Christlich-Sozialer Union (CSU) und Christlich-Demokratischer Union (CDU) unterschieden wird. Die Parteien stehen sich programmatisch sehr nahe und bilden auf Bundesebene eine Fraktionsgemeinschaft. Hinzu kommt, dass die CSU nur zu Wahlen in Bayern antritt, während die CDU nur in den übrigen Ländern aktiv ist. Die Regierungszeiten der unterschiedlichen Politikkonstellationen auf Länderebene sowie die Behandlung der oben erwähnten Ausnahmefälle werden im Anhang B erläutert.

Tabelle 1 : Berücksichtigte Politikkonstellationen

\begin{tabular}{|l|l|}
\hline Dummyvariable & Politikkonstellation (Parteien) \\
\hline SPD (Referenzkategorie) & Sozialdemokratische Partei Deutschlands (SPD) \\
SPD-GR & SPD, Bündnis 90/Die Grünen (Grüne) \\
SPD-CDU & SPD, Christlich-Demokratische Union (CDU) \\
SPD-FDP & SPD, Freiheitlich-Demokratische Partei Deutschlands (FDP) \\
CDU & CDU bzw. Christlich-Soziale Union (CSU) \\
CDU-FDP & CDU, FDP \\
\hline
\end{tabular}

\footnotetext{
${ }^{2}$ Wir danken Helge Berger, Freie Universität Berlin, für die Bereitstellung der Politik-Daten.
} 


\section{Wirtschaftliche Strukturvariablen}

Als Kontrollvariablen berücksichtigen wir einige wirtschaftliche Strukturvariablen. Dazu zählen die Arbeitslosenquote, das durchschnittliche Arbeitnehmerentgelt und die Schuldenhöhe pro Einwohner, jeweils bezogen auf das einzelne Bundesland. Durch diese Variablen soll berücksichtigt werden, dass die Hochschulausgaben der Länder auch etwa von der allgemeinen Arbeitsmarktlage, dem wirtschaftlichen Entwicklungsstand, oder der fiskalischen Situation des jeweiligen Bundeslandes abhängen können (vgl. dazu auch Seitz und Baum 2003). Die Arbeitslosenquote wird einerseits in das Modell eingeführt, um auf die Ansprüche zu kontrollieren, die von den Ländern an das Bildungssystem gestellt werden. Es erscheint allerdings auch möglich, dass aufgrund von hoher Arbeitslosigkeit auf Länderebene ein Verdrängungswettbewerb bezüglich der öffentlichen Ausgaben eintreten kann. Das Arbeitnehmerentgelt dient als Maßgröße für die tax efficiency der Länder, der Einfluss der Entgelte auf die Hochschulausgaben kann aber auch über politökonomische Argumente hergestellt werden (vgl. Abschnitt 2.1). Sowohl Arbeitslosenquote als auch die Entgelte können zudem Maßgrößen darstellen, die Informationen über den Ertrag der Hochschulbildung liefern und damit ebenfalls über den politischen Prozess auf die Hochschulausgaben einwirken. Über die Einführung der mit der Einwohnerzahl skalierten Schuldenhöhe eines Bundeslandes soll schließlich darauf kontrolliert werden, dass Bundesländer, die unter einer hohen finanziellen Belastung stehen, geringere Pro-Kopf-Mittel für die Hochschulausbildung zur Verfügung stellen können. Deskriptive Statistiken dieser Variablen sind in Tab. A1 im Anhang enthalten.

\subsection{Schätzansatz}

Die Schätzungen des Zusammenhangs zwischen Hochschulausgaben, demographischen und Politikvariablen basieren auf einem Panel der zehn westdeutschen Bundesländer (ohne Berlin) im Zeitraum zwischen 1985 und 2002. Ein Vorteil der Panelschätzung besteht in der gleichzeitigen Nutzung der Querschnittsvariation über die Länder und der Zeitreihenvariation. Ein weiterer Vorteil der Panelschätzung besteht im Vergleich zu Zeitreihenschätzungen für einzelne Länder in der größeren Anzahl an Beobachtungen und der - je nach dem verwendeten Panelschätzer - mehr oder weniger präzise geschätzten Modellparametern. Die üblichen (statischen) Panelschätzer sind Pooled OLS, der Fixed-Effects und der RandomEffects Schätzer. Allerdings setzen diese Schätzmethoden Homogenität der Steigungskoeffizienten zwischen den Beobachtungseinheiten voraus, d.h. die Koeffizienten der erklärenden Variablen dürfen nicht zwischen den Ländern variieren. 
In einem ersten Schritt haben wir daher diese Homogenitätsannahme hinsichtlich der hier verwendeten Demographie-Variablen sowie des Zeittrends mittels einfachen Zeitreihenschätzungen für die einzelnen Länder überprüft. ${ }^{3}$ Dabei zeigte sich, dass sich bei der Verwendung des Anteils der über 55-jährigen als Demographie-Variable die Konfidenzintervalle der geschätzten Koeffizienten dieser Variablen für alle 10 Bundesländer überlappen. Auch konnten wir in dieser Spezifikation keine unterschiedlichen Trendeffekte feststellen. Hingegen ergaben die Zeitreihenschätzungen mit dem Anteil der über 65-jährigen als Demographie-Variable, dass sich sowohl deren geschätzter Koeffizient als auch der für den Zeittrend im Bundesland Niedersachsen signifikant von den jeweils für die anderen Bundesländer geschätzten Koeffizienten signifikant unterscheiden. Diese Unterschiede werden bei den Panelschätzungen durch die Aufnahme von Interaktionsdummies zwischen einem Bundesland-Dummy für Niedersachsen und dem Anteil der über 65-jährigen bzw. dem Zeittrend berücksichtigt.

Die Zeitreihenschätzungen für die einzelnen Bundesländer weisen auf starke Autokorrelation in den Residuen hin. Ein Grund dafür könnte in verzögerter Anpassung in den Hochschulausgaben auf Änderungen der erklärenden Variablen bestehen. Dies würde für eine dynamische Spezifikation der Schätzgleichungen mit verzögerten Werten der Hochschulausgaben als erklärende Variable und der Schätzung mittels der verallgemeinerten Momenten-Methode sprechen (vgl. z.B. Greene, 2003, Kap. 13.6). Da die statistischen Eigenschaften dieser Schätzer für unsere Anwendung aufgrund der geringen Anzahl an Beobachtungen sowohl über die Zeit (18) als vor allem auch im Querschnitt (10) unbekannt sind, verzichten wir hier auf eine explizit dynamische Modellierung und führen statt dessen eine Autokorrelationsbereinigung der Residuen aus den im Prinzip statischen Panelschätzungen durch (vgl. z.B. Greene 2003, Kap. 13.7.3).

\subsection{Schätzergebnisse}

Die Schätzergebnisse auf Basis von Pooled OLS, Fixed sowie Random Effects (FE / RE), sowohl ohne als auch mit Autokorrelationskorrektur, sind in Tabelle A2 im Anhang für die beiden unterschiedlichen Spezifikationen der Demographie-Variablen zusammengefasst. Zusätzlich zu den erwähnten Kontrollvariablen enthalten alle Spezifikationen einen linearen Trendterm. Die Effekte der neben den Demographie- und Politikvariablen aufgenommenen wirtschaftlichen Strukturvariablen sind in den meisten Spezifikationen statistisch insignifikant. Für diesen Fall (10\%-Level) werden diese Variablen jeweils ausgeschlossen. In den Pooled OLS-Schätzungen haben wir außerdem eine Dummy-Variable für die beiden

\footnotetext{
${ }^{3}$ Die Ergebnisse dieser Zeitreihenschätzungen sind von den Autoren auf Anfrage erhältlich.
} 
Stadtstaaten Bremen und Hamburg aufgenommen, um strukturelle Unterschiede bei den Hochschulausgaben zwischen diesen und den Flächenländern zu berücksichtigen (siehe Abschnitt 3.1.). Da diese Variable über die Zeit nicht variiert, kann für sie bei FE kein Koeffizient geschätzt werden; ihr Einfluss wird aber wie bei RE durch die Ländereffekte miterfasst. Tabelle A3 enthält die Ergebnisse der üblichen Test auf die Existenz fixer (F-Test) bzw. zufälliger Ländereffekte (LM-Test) sowie des Hausman-Tests auf Korrelation zwischen diesen und den erklärenden Variablen des Modells (vgl. z.B. Greene, 2003, Kap. 13.3 und 13.4). Zudem haben wir die Residuen der Regressionen auf Autokorrelation 1. und 2. Ordnung hin untersucht. Die Resultate finden sich in Tabelle A4.

Die Ergebnisse dieser Tests weisen in allen Spezifikationen auf Autokorrelation 1. Ordnung in den Residuen und auf signifikante Ländereffekte hin, die mit den erklärenden Modellvariablen korreliert sind. Daher liefern nur die FE-Schätzungen unverzerrte - und nach Autokorrelationsbereinigung - effiziente Schätzungen der Koeffizienten der Demographie- und Politikvariablen. Die Ergebnisse der autokorrelationsbereinigten FESchätzungen sind für die beiden Spezifikationen der Demographie-Variablen in Tabelle 2 ausgewiesen.

Tabelle 2: Panelschätzungen des Einflusses der Demographie und politischer Konstellationen auf die Hochschulausgaben der Länder - FE-Schätzungen mit Autokorrelationsbereinigung

\begin{tabular}{|l|r|r|r|r|r|r|}
\hline & Demographie & $\begin{array}{l}\text { SPD- } \\
\text { GR }\end{array}$ & $\begin{array}{l}\text { SPD- } \\
\text { FDP }\end{array}$ & $\begin{array}{l}\text { SPD- } \\
\text { CDU }\end{array}$ & $\begin{array}{l}\text { CDU- } \\
\text { FDP }\end{array}$ & CDU \\
\hline $\begin{array}{l}\text { über 65- } \\
\text { jährige }\end{array}$ & $\begin{array}{r}-0,11 \\
(-0,30)\end{array}$ & $\begin{array}{r}0,02 \\
(1,35)\end{array}$ & $\begin{array}{r}0,01 \\
(0,35)\end{array}$ & $\begin{array}{r}0,08^{*} \\
(1,65)\end{array}$ & $\begin{array}{r}0,05^{* *} \\
(2,40)\end{array}$ & 0,02 \\
& & & & & & \\
\hline $\begin{array}{l}\text { über 55- } \\
\text { jährige }\end{array}$ & $\begin{array}{r}-1,22^{* *} \\
(-1,98)\end{array}$ & $\begin{array}{r}0,02 \\
(0,98)\end{array}$ & $\begin{array}{r}0,01 \\
(0,43)\end{array}$ & $\begin{array}{r}0,06 \\
(1,41)\end{array}$ & $\begin{array}{r}0,04^{*} \\
(1,66)\end{array}$ & $\begin{array}{r}0,02 \\
(0,80)\end{array}$ \\
& & & & & & \\
\hline
\end{tabular}

Anmerkungen: t-Werte in Klammern. * und ** stehen für Signifikanz auf Zehn- bzw. Fünf-Prozent-Niveau; die Schätzungen enthalten die oben erwähnten Kontrollvariablen; in der Spezifikation mit Anteil der über 65jährigen ist die Schuldengröße, mit Anteil der über 55-jährigen zudem die Arbeitslosenquote wegen. Insignifikanz ausgeschlossen. Die detaillierten Schätzergebnisse mit statistischen Tests und Maßzahlen sind in den Tabellen A2 - A4 ausgewiesen.

Der geschätzte Einfluss der Bevölkerungsalterung auf die Hochschulausgaben der Länder weist hier in beiden Ansätzen das erwartete negative Vorzeichen auf. Wird die Bevölkerungsalterung durch den Anteil der über 65-jährigen abgebildet, ist der geschätzte Einfluss der Demographie auf die Hochschulausgaben zwar leicht negativ, aber statistisch nicht signifikant. Die Schätzung der gleichen Spezifikation unter Einbezug der 55- bis 65- 
jährigen in die „Altenquote“ liefert stärkere Anzeichen auf intergenerationelle Konflikte bezüglich der Hochschulausgaben. Der geschätzte Koeffizient der Demographie-Variable ist in dieser Spezifikation auf dem 5\%-Niveau statistisch signifikant, die geschätzte Elastizität liegt bei ca. -1,2: eine einprozentige Zunahme der über 55-jährigen Bevölkerung eines Bundeslandes führt demnach zu eine Abnahme der Hochschulausgaben um mehr als ein Prozent. ${ }^{4}$

Die geschätzten Parteieneffekte auf die Hochschulausgaben der Länder sind als Abweichung in Prozent $(\Delta \%)$ von den Pro-Kopf-Hochschulausgaben einer SPDAlleinregierung im jeweiligen Bundesland, die als Referenzkategorie definiert wurde, zu interpretieren ${ }^{5}$. Die Schätzergebnisse implizieren, dass die Hochschulausgaben der Länder im Beobachtungszeitraum unter einer Großen Koalition um acht Prozent (bei Verwendung der über 65-jährigen als Demographie-Variable) bzw. sechs Prozent. (über 55-jährige) höher ausgefallen sind als unter einer SPD-Alleinregierung. Die Hochschulausgaben unter CDUFDP Landesregierungen fallen ebenfalls höher aus als unter einer SPD Alleinregierung (um fünf bzw. vier Prozent). Für CDU-Alleinregierungen ergeben unsere Schätzungen etwas kleinere (zwei Prozent) und statistisch insignifikante Effekte. Die Hochschulausgaben pro Kopf unterscheiden sich daher unter CDU- und SPD Alleinregierungen statistisch nicht signifikant voneinander.

Bemerkenswert erscheint, dass die geschätzten Effekte der Politikvariablen sehr robust bezüglich alternativer Schätzverfahren sowie Spezifikationen der Demographie-Variablen sind (vgl. Ergebnisse in Tabelle A2 im Anhang). Bei allen konsistenten Schätzungen kann unabhängig von der Definition der Demographie-Variablen die Nullhypothese abgelehnt werden, dass die Variablen SPD-CDU, CDU-FDP und $C D U$ gemeinsam die Hochschulausgaben nicht beeinflussen, während sich linksorientierte Koalitionen (Rot-Grün sowie Sozial-Liberal) in dieser Hinsicht kaum von SPD-Alleinregierungen unterscheiden. Ansonsten gilt nach dieser Analyse für die Hochschulausgaben jedoch policy matters.

Von den Kontrollvariablen geht lediglich die Entgeltvariable mit hoher Robustheit bezüglich der verschiedenen Modellvariationen signifikant in die Schätzgleichung ein. Die Elastizitäten liegen in den konsistenten und effizienten FE-Cochrane-Orcutt-Schätzungen bei 0,55 bzw. 0,57 . Dies könnte beispielsweise auf einen positiven Effekt des Ertrags von Bildung auf die Hochschulausgaben hindeuten (vgl. Abschnitt 3.2.). Die Schuldengröße, ein

\footnotetext{
4 Da die Hochschulausgaben und die Demographie-Variablen mit logarithmierten Werten in die Schätzung eingehen, gibt der geschätzte Koeffizient der Demographie-Variablen direkt die erwähnte Elastizität an.

5 Formal gilt: $\Delta \%=\left(\exp \left(\hat{\beta}_{j}\right)-1\right) * 100$, mit $\hat{\beta}_{\mathrm{j}}:=$ geschätzter Koeffizient der Politik-Variablen $j$.

6 Die Koeffizienten sind auf 10- bzw. 5\%-Level signifikant von Null verschieden.
} 
linearer Trendterm sowie die Arbeitslosenquote als weitere mögliche Determinanten der Hochschulausgaben ergeben betragsmäßig kleinere Koeffizienten, die zudem über die verwendeten Spezifikationen bzgl. Größenordnung und Signifikanz stark schwanken. Über ihren Einfluss auf die Hochschulausgaben können daher in dieser Arbeit kaum verlässliche Schlussfolgerungen gezogen werden.

\section{Zusammenfassung und wirtschaftspolitische Schlussfolgerungen}

Wir haben auf der Basis von Paneldaten für die westdeutschen Bundesländer untersucht, ob und in welchem Maße der demographische Wandel sowie die unterschiedlichen politischen Konstellationen auf die Pro-Kopf-Hochschulausgaben auf Länderebene einwirken. Je älter eine Person, desto kürzer der Zeitraum, in dem sie von den Hochschulausgaben profitieren kann. Daher kann ein negativer Zusammenhang zwischen Alterung der Gesellschaft und den Hochschulausgaben erwartet werden. Die Partisanentheorie impliziert, dass linke Parteien aus ideologischen Gründen höhere Staatsausgaben befürworten als rechte. Ob diese Hypothesen die Entwicklung der Hochschulausgaben in Deutschland erklären können, wurde bisher für Deutschland noch nicht empirisch untersucht. Unsere Untersuchung leistet dazu einen ersten Beitrag.

Bezüglich der Analyse des Einflusses des demographischen Wandels sind die Ergebnisse ambivalent. Während in der Untersuchung für den Anteil der über 65-jährigen keine Auswirkungen auf die Hochschulausgaben ermittelt werden können, lässt sich für den Anteil der über 55-jährigen ein relativ starker Effekt empirisch nachweisen. Der Grund für den stärkeren Effekt der über 55-jährigen dürfte hauptsächlich in der größeren politischen Macht dieser Altersgruppe liegen. Während diese Gruppe im Beobachtungszeitraum teilweise die 30-Prozent-Marke am Anteil der Gesamtbevölkerung überschreitet, liegen die über 65jährigen stets unterhalb von 20 Prozent. Die Tatsache, dass die Hochschulausgaben stärker mit den über 55- als den 65-jährigen variieren, kann hingegen nicht damit erklärt werden, dass 55 Jahre die Grenze zwischen Befürwortern und Gegnern von Investitionen in das Hochschulsystem besser widerspiegelt. Dieser Übergang verläuft fließend, so dass letztendlich jegliche Altersgrenze willkürlich gesetzt ist. Jedoch erzwingt nur eine starke Wählergruppe mit ähnlichen Interessen die Durchsetzung der von ihr präferierten Politik und diese Voraussetzung wird durch die Wahl der über 55-jährigen eben eher erfüllt als durch die über 65-jährigen. Dieser Effekt überkompensiert dabei denjenigen, dass theoriegemäß gerade die 55- bis 65-jährigen den Hochschulausgaben weniger abgeneigt sein müssten als etwa 70- oder 75-jährige. Je jünger eine Person, desto höhere Hochschulausgaben wird sie tendenziell befürworten, wie dies auch dem Ressourcenpool-Argument entspricht. In jedem 
Falle suggerieren unsere Ergebnisse, dass in Deutschland gewisse intergenerationelle Verteilungskonflikte bezüglich der Hochschulausgaben bestehen.

Auch identifiziert diese Arbeit Auswirkungen politischer Konstellationen auf die Hochschulausgaben in Deutschland. Die Hochschulausgaben der Bundesländer variieren teilweise erheblich mit der regierenden Partei oder Koalition. Allerdings entsprechen unsere Ergebnisse nicht der klassischen Partisanentheorie, nach der Parteien des linken Spektrums zu höheren Staatsausgaben neigen. Nach den Ergebnissen unserer Studie zeichnen sich bürgerliche Konstellationen sowie die Große Koalition für das höchste Niveau der Hochschulausgaben verantwortlich. Hauptgrund hierfür könnte Klientelpolitik der Parteien sein, wenn man unterstellt, dass etwa bürgerliche Parteien ihre Wählerschaft vornehmlich aus den Schichten rekrutieren, die tendenziell hohe Hochschulausgaben unterstützen.

Die aufgezeigte Existenz von intergenerationellen Verteilungskonflikten weist darauf hin, dass auf Grund der zu erwartenden Zunahme des Anteils der über 55-jährigen in Deutschland eine deutliche Kürzung der Hochschulausgaben in der Zukunft wahrscheinlich ist. Dies geht tendenziell einher mit den jüngsten Anstrengungen vieler Bundesländer, die Kosten für die Hochschulausgaben stärker vom eigenen Haushalt auf andere Köpfe zu verteilen. Über Studiengebühren wird einerseits die Studentenschaft stärker in die finanzielle Verantwortung gezogen. Auf der anderen Seite nimmt der Druck auf die Hochschulen zu, verstärkt Gelder einzuwerben, um Mittelkürzungen vermeiden zu können. Auch wenn diese Vorgänge sicherlich weitere Gründe als nur die Alterung der Gesellschaft haben mögen: Die Zunahme der Älteren hat einen negativen Effekt auf die Hochschulausgaben, und dieser existiert, obwohl weder ein Rückgang der Studentenzahlen noch ein generell geringerer Bedarf an höherer Ausbildung und Forschung festzustellen ist. Die Jungen sowie die zukünftigen Generationen könnten die negativen Konsequenzen von rückläufigen Hochschulausgaben zu spüren bekommen, sollte der Rückgang mit einem Verlust an Qualität der Hochschulbildung einhergehen. Diesbezüglich ist jedoch sicherlich weitergehende Forschung von Nöten.

Die Existenz von Parteieneffekten weist darauf hin, dass nicht nur fundamentale wirtschaftliche Einflussfaktoren eine Rolle bei den Hochschulausgaben spielen. Auf der einen Seite kann man dies bedauern, da dieses Resultat politische Zyklen in den deutschen Hochschulausgaben andeutet. Die Hochschulausgaben sind damit subjektiven Zielen von Parteien und Regierungen unterworfen. Andererseits jedoch impliziert diese Erkenntnis auch, dass die Bürger eine echte Wahl besitzen, da keine Politikkonvergenz bezüglich der Hochschulausgaben erreicht wird. 


\section{Literaturverzeichnis}

Alesina, A. (1988), Credibility and Policy Convergence in a Two-Party-System with Rational Voters, American Economic Review 78, 796-805.

Alt, J. und R. Lowry (1994), Divided Government, Fiscal Institutions, and Budget Deficits: Evidence from the States, American Political Science Review 88, 811-828.

Baum, B. und H. Seitz (2003), Demographischer Wandel und Bildungsausgaben: Empirische Evidenz für die westdeutschen Länder, Vierteljahreshefte zur Wirtschaftsforschung 72, S. 205-219.

Blais, A., D. Blake und S. Dion (1993), Do Parties Make a Difference? Parties and the Size of Government in Liberal Democracies, American Journal of Political Science 37, 40-62.

Boix, C. (1997), Political Parties and the Supply Side of the Economy: The Provision of Physical and Human Capital in Advanced Economies 1960-90, American Journal of Political Science 41, 814-845.

Borck, R. (2006), Central versus Local Education Finance: A Political Economy Approach, DIW Discussion Papers No. 565/2006, Berlin.

Cusack, T. (1997), Partisan Politics and Public Finance: Changes in Public Spending in the Industrialized Democracies 1955-1989, Public Choice 91, 375-395.

Downs, A. (1957), An Economic Theory of Democracy, New York.

Epple, D. und R. Romano (1996), Ends Against the Middle: Determining Public Service Provision when there Are Private Alternatives, Journal of Public Economics 62, $297-$ 325.

Fernandez, R. und R. Rogerson (2001), The Determinants of Public Education Expenditures: Longer-Run-Evidence from the States, Journal of Education Finance 27, 567-584.

Gradstein, M. und M. Kaganovich (2004), Aging Population and Education Finance, Journal of Public Economics 88, 2469-2485.

Greene, W. (2003), Econometric Analysis, International Edition, 5. Auflage, Upper Saddle River.

Harris, A., W. Evans und R. Schwab (2001), Education Spending in an Aging America, Journal of Public Economics 81, 449-472.

Haupt, A. und E. Janeba (2003), Bildung im Zeitalter mobilen Humankapitals, Vierteljahreshefte zur Wirtschaftsforschung 72, 173-187.

Hetmeier, H. (2000), Bildungsausgaben im Vergleich, Zeitschrift für Pädagogik 46/1, 19-38.

Hetmeier, H. und M. Weiß (2001), Bildungsausgaben, in: Böttcher, W., K. Klemm und T. Rauschenbach (Hrsg.), Bildung und Soziales in Zahlen, Weinheim und München, 39-56.

Hibbs, D. (1977), Political Parties and Macroeconomic Policy, The American Political Science Review 71, 1467-1487.

Homburg, S. (1994), Anreizwirkungen des deutschen Finanzausgleichs, Finanzarchiv 51, 312-330.

Jochimsen, B. und R. Nuscheler (2004), The Political Economy of the German Länder Deficit, mimeo, Wissenschaftszentrum Berlin.

Kemnitz, A. (2000), Bildungspolitik in alternden Gesellschaften, Aachen. 
Kemnitz, A. und R. von Weizsäcker (2003), Bildungsreform in der Demokratie, Vierteljahreshefte zur Wirtschaftsforschung 72, 188-204.

Ladd, H. und S. Murray (2001), Intergenerational Conflict Reconsidered: County Demographic Structure and the Demand for Public Education, Economics of Education Review 20, 343-357.

Olsen, M. (1965), The Logic of Collective Action, Cambridge.

Poterba, J. (1997), Demographic Structure and the Political Economy of Public Education, Journal of Policy Analysis and Management 16, 48-66.

Poterba, J. (1998), Demographic Change, Intergenerational Linkages, and Public Education, American Economic Association Papers and Proceedings 88, 315-320.

Rodden, J. (2001), And the Last Shall be First: Federalism and Fiscal Outcomes in Germany, Massachusetts Institute of Technology, mimeo.

Roubini, N. und J. Sachs (1989), Government Spending and Budget Deficits in the Industrial Countries, Economic Policy 12, 100-132.

Schmidt, M. (2003), Ausgaben für Bildung im internationalen Vergleich, Aus Politik und Zeitgeschichte 21-22/2003, 6-11.

Seitz, H. (2000), Fiscal Policy, Deficits and Politics of Subnational Governments: The Case of the German Laender, Public Choice 102, 183-218.

Seitz, H., D. Freigang und G. Kempkes (2005), Demographic Change and Federal Systems, in: Färber, G. und N. Otter (Hrsg.), Spatial Aspects of Federative Systems, Speyerer Forschungsbericht 242, Speyer, 223-251.

Sinn, H.-W. und S. Uebelmesser (2003), Pensions and the Path to Gerontocracy in Germany, European Journal of Political Economy 19 / 1, 153-158

Statistisches Bundesamt (Hrsg.) (2003), Bericht zur finanziellen Lage der Hochschulen, Wiesbaden.

Steiner, V. (2002), Intertemporale Effekte der Bildungspolitik, in: Theurl, E., H. Winner und R. Sausgruber (Hrsg.), Kompendium der österreichischen Finanzpolitik, Berlin, 561-596. 


\section{Anhang}

\section{A. Konstruktion der Hochschulausgaben}

Die Hochschulausgaben werden im Zweifel institutionell abgegrenzt. „Bestehen zum Beispiel rechtlich selbständige Institute an Hochschulen oder haben diese den Charakter einer selbständigen Einrichtung (z.B. das Institut für Weltwirtschaft an der Universität Kiel), so werden deren Ausgaben nicht in den Hochschulbereich einbezogen“ (Hetmeier, 2000, S. 30). Gleiches gilt für Gärten, Museen und Bibliotheken, die nicht direkter Bestandteil einer Hochschule sind. Ausgaben von Ämtern, die außerhalb der Hochschulen deren Verwaltungsaufgaben wahrnehmen, werden wie der Schuldendienst für Hochschulinvestitionen ebenfalls den Hochschulausgaben nicht zugerechnet. Unberücksichtigt bleiben weiterhin die Ausgaben der akademischen Lehrkrankenhäuser. Nicht enthalten sind zudem Zahlungen, die den Lebensunterhalt von Studierenden betreffen (wie bspw. Stipendien). Schließlich sind die Grundmittel bereinigt um die Ausgaben für die Drittmittelforschung und die Ausgaben für Leistungen, die nicht für Lehre und Forschung bestimmt sind. Sie enthalten außerdem keine Zuschläge für die Altersversorgung und die Krankenbehandlung der Beamten des Hochschulbereichs. Im Haushaltswesen der Hochschulen nicht erfasste Mittel der Deutschen Forschungsgemeinschaft (DFG) wie zur Doktoranden- und Postdoktorandenförderung, die die DFG unmittelbar für den Hochschulbereich tätigt, zählen hingegen zu den Hochschulausgaben (vgl. Hetmeier, 2000).

\section{B. Politikkonstellationen}

In Bayern regierte im Beobachtungszeitraum durchgängig die Christlich-Soziale Union (CSU) ohne Unterstützung eines Koalitionspartners. In Baden-Württemberg folgte eine Große Koalition (SPD, CDU) zwischen 1992 und 1995 auf eine CDU-Alleinregierung. Ab 1996 bildeten CDU und FDP eine Koalition. Bremen wurde bis 1991 von der SPD alleine regiert. Es folgte eine dreijährige Koalition von SPD, FDP und Grünen, die in dieser Arbeit als SPDFDP-Koalition gezählt wird, da die FDP mehr Sitze in der Bremer Bürgerschaft hielt als die Grünen, sowie anschließend eine Große Koalition. In Hamburg folgte 1987 ein SozialLiberales Bündnis (SPD-FDP) auf eine SPD-Alleinregierung. Zwischen 1991 und 1993 regierte wiederum die SPD alleine. Von 1993 bis 1997 war die Statt-Partei Koalitionspartner der SPD. Diese Phase wird in dieser Arbeit als SPD-Alleinregierung behandelt. Ab 1998 regierte die SPD mit den Grünen, bis 2002 eine Koalition aus CDU, FDP und der SchillPartei an die Macht kam. Diese Amtsperiode wird als CDU-FDP-Koalition gewertet.

Nach einer SPD-Alleinregierung übernahm Rot-Grün (SPD und Grüne) 1986 für ein Jahr die Macht in Hessen. Es folgten vier Jahre CDU-Alleinregierung, darauf nochmals acht Jahre Rot-Grün und schließlich eine Koalition aus CDU und FDP. In Niedersachsen übernahm 1986 für vier Jahre eine Schwarz-Gelbe Koalition (CDU-FDP) von der CDU die Regierungsgeschäfte. Anschließend regierte Rot-Grün vier Jahre lang. Es folgte eine SPDAlleinregierung. In Nordrhein-Westfalen erfolgte im Beobachtungszeitraum nur ein Regierungswechsel: 1995 ging die SPD-Alleinregierung in ein Rot-Grünes Bündnis über. In Rheinland-Pfalz herrschte die CDU zunächst für ein Jahr alleine und anschließend für vier Jahre in einer Koalition mit der FDP, bevor eine Sozial-Liberale Koalition (SPD-FDP) die Macht übernahm. Schleswig-Holstein kam im Anschluss an eine CDU-Alleinregierung 1988 die SPD für acht Jahre an die Macht. Ab 1996 stellte die SPD bis zum Ende des Beobachtungszeitraums zusammen mit den Grünen das Kabinett. Im Saarland löste im Jahr 2000 die CDU die SPD als Regierungspartei ab. Umgekehrt lösten die Sozialdemokraten in 
Schleswig-Holstein 1988 die Union ab, bevor 1996 ein Rot-Grünes Bündnis die an die Regierung kam. 


\section{Deskriptive Statistiken}

\section{Tabelle A1: Deskriptive Statistiken}

\begin{tabular}{|c|c|c|c|c|c|c|c|c|c|c|c|c|c|}
\hline \multirow[t]{2}{*}{ Bundesland } & \multirow[t]{2}{*}{$\mathbf{T}$} & \multicolumn{2}{|c|}{$\begin{array}{l}\text { Hochschul- } \\
\text { ausgaben pro Kopf } \\
\text { (in Euro) }\end{array}$} & \multicolumn{2}{|c|}{$\begin{array}{c}\text { Anteil der über } \\
\text { 65-jährigen an der } \\
\text { Gesamtbevölkerung } \\
\text { (in \%) }\end{array}$} & \multicolumn{2}{|c|}{$\begin{array}{c}\text { Anteil der über } \\
\text { 55-jährigen an der } \\
\text { Gesamtbevölkerung } \\
\text { (in \%) }\end{array}$} & \multicolumn{2}{|c|}{$\begin{array}{l}\text { Arbeitslosenquote } \\
\text { (in \%) }\end{array}$} & \multicolumn{2}{|c|}{$\begin{array}{l}\text { Durchschnittliches } \\
\text { Arbeitnehmerentgelt } \\
\text { (Euro, in Preisen } \\
\text { von 1996) }\end{array}$} & \multicolumn{2}{|c|}{$\begin{array}{c}\text { Schulden pro Kopf } \\
\text { (Euro, in Preisen } \\
\text { von 1996) }\end{array}$} \\
\hline & & MW & SA & MW & SA & MW & SA & MW & SA & MW & SA & MW & SA \\
\hline Bayern & 18 & 165,82 & 15,29 & 0,15 & 0,01 & 0,27 & 0,01 & 8,34 & 1,45 & 30182,55 & 1860,04 & 1444,89 & 81,49 \\
\hline Baden-Württemberg & 18 & 187,02 & 14,41 & 0,15 & 0,01 & 0,27 & 0,01 & 6,01 & 1,49 & 31436,37 & 1653,40 & 2430,50 & 284,80 \\
\hline Bremen & 18 & 244,83 & 57,27 & 0,18 & 0,00 & 0,30 & 0,01 & 14,36 & 1,63 & 31485,79 & 1460,80 & 12525,18 & 1000,25 \\
\hline Hessen & 18 & 182,11 & 8,80 & 0,16 & 0,01 & 0,28 & 0,01 & 8,11 & 1,65 & 31864,80 & 1744,88 & 3080,16 & 387,50 \\
\hline Hamburg & 18 & 294,64 & 20,73 & 0,17 & 0,01 & 0,30 & 0,01 & 11,46 & 1,82 & 33056,10 & 1625,92 & 7761,17 & 1296,27 \\
\hline Nordrhein-Westfalen & 18 & 160,06 & 10,75 & 0,16 & 0,01 & 0,28 & 0,01 & 10,84 & 1,30 & 31171,94 & 1296,65 & 3638,92 & 462,58 \\
\hline Niedersachsen & 18 & 157,76 & 16,68 & 0,16 & 0,01 & 0,28 & 0,01 & 11,95 & 1,70 & 29288,71 & 1197,99 & 3637,05 & 590,60 \\
\hline Rheinland-Pfalz & 18 & 120,00 & 16,74 & 0,16 & 0,01 & 0,29 & 0,01 & 9,01 & 1,55 & 29364,07 & 1390,69 & 3710,22 & 593,95 \\
\hline Schleswig-Holstein & 18 & 145,48 & 11,59 & 0,16 & 0,01 & 0,28 & 0,02 & 10,89 & 1,49 & 27966,27 & 1413,11 & 4684,29 & 674,14 \\
\hline Saarland & 18 & 171,37 & 11,30 & 0,17 & 0,01 & 0,29 & 0,02 & 12,11 & 1,71 & 29392,22 & 1279,36 & 6050,96 & 716,14 \\
\hline $\operatorname{Gesamt}(\mathrm{N} \times \mathrm{T})$ & 180 & 182,91 & 53,21 & 0,16 & 0,01 & 0,28 & 0,02 & 10,31 & 2,78 & 30520,88 & 2070,61 & 4896,34 & 3142,18 \\
\hline
\end{tabular}

Anmerkungen: T: Anzahl der Beobachtungen (Jahre), MW: Mittelwert, SA: Standardabweichung; die monetären Variablen sind in Euro in Preisen von 1996 angegeben 


\section{Schätzergebnisse}

Tabelle A2: Panelschätzungen des Einflusses der Demographie und politischer Konstellationen auf die Hochschulausgaben der Länder

\begin{tabular}{|c|c|c|c|c|c|c|c|c|c|c|c|c|}
\hline & \multicolumn{2}{|c|}{ Pooled OLS } & \multicolumn{2}{|c|}{ Pooled Cochrane-Orcutt } & \multicolumn{2}{|c|}{ Fixed Effects } & \multicolumn{2}{|c|}{$\begin{array}{c}\text { Fixed Effects } \\
\text { Cochrane-Orcutt }\end{array}$} & \multicolumn{2}{|c|}{ Random Effects } & \multicolumn{2}{|c|}{$\begin{array}{l}\text { Random Effects } \\
\text { Cochrane-Orcutt }\end{array}$} \\
\hline & $>65$ Jahre & >55 Jahre & $>65$ Jahre & >55 Jahre & $>65$ Jahre & >55 Jahre & $>65$ Jahre & >55 Jahre & $>65$ Jahre & $>55$ Jahre & $>65$ Jahre & >55 Jahre \\
\hline \multirow{2}{*}{ Demographie } & $-0,10$ & $-0,13$ & 0,08 & $-0,87 * *$ & $-0,47 * *$ & 0,21 & $-0,11$ & $-1,22 * *$ & $-0,20$ & 0,43 & $0,82 * *$ & $-1,01 *$ \\
\hline & $(-0,54)$ & $(-0,44)$ & $(0,25)$ & $(-2,45)$ & $(-2,33)$ & $(0,74)$ & $(-0,30)$ & $(-1,98)$ & $(-0,92)$ & $(1,17)$ & $(2,39)$ & $(-1,88)$ \\
\hline \multirow[t]{2}{*}{ SPD-GR } & $-0,02$ & $-0,03$ & 0,03 & 0,02 & 0,02 & $-0,004$ & 0,02 & 0,02 & & & & \\
\hline & $(-1,08)$ & $(-1,58)$ & $(1,44)$ & $(1,01)$ & $(0,64)$ & $(-0,23)$ & $(1,35)$ & $(0,98)$ & & & & \\
\hline \multirow[t]{2}{*}{ SPD-FDP } & $-0,21 * * *$ & $-0,22 * *$ & $-0,04$ & $-0,04$ & 0,02 & $-0,01$ & 0,01 & 0,01 & v & $\sim$ & $\sim$ & $\sim$ \\
\hline & $(-4,27)$ & $(-4,04)$ & $(-1,13)$ & $(-1,21)$ & $(0,56)$ & $(-0,40)$ & $(0,35)$ & $(0,43)$ & & & & \\
\hline \multirow[t]{2}{*}{ SPD-CDU } & 0,03 & 0,03 & 0,05 & 0,04 & $0,15^{* * *}$ & $0,16^{* * *}$ & $0,08^{*}$ & 0,06 & $0,17 * * *$ & $0,18 * * *$ & 0,02 & $0,05^{*}$ \\
\hline & $(0,60)$ & $(0,67)$ & $(0,96)$ & $(0,89)$ & $(2,89)$ & $(3,49)$ & $(1,65)$ & $(1,41)$ & $(3,40)$ & $(5,76)$ & $(1,36)$ & $(1,87)$ \\
\hline \multirow[t]{2}{*}{ CDU-FDP } & 0,0007 & $-0,006$ & $0,05 * *$ & $0,04^{*}$ & $0,07^{*}$ & $0,05^{* *}$ & $0,05 * *$ & $0,04^{*}$ & $0,07^{* * *}$ & $0,08 * * *$ & 0,02 & $0,03 *$ \\
\hline & $(0,03)$ & $(-0,34)$ & $(2,44)$ & $(1,91)$ & $(1,84)$ & $(2,39)$ & $(2,40)$ & $(1,66)$ & $(2,91)$ & $(3,42)$ & $(1,48)$ & $(1,96)$ \\
\hline \multirow[t]{2}{*}{ CDU } & 0,003 & $-0,005$ & $0,05^{* *}$ & $0,04 * *$ & $-0,07$ & $-0,06$ & 0,02 & 0,02 & 0,01 & $0,09 * * *$ & 0,05 & 0,03 \\
\hline & $(0,11)$ & $(-0,22)$ & $(2,00)$ & $(2,05)$ & $(-0,30)$ & $(-0,27)$ & $(0,90)$ & $(0,80)$ & $(0,36)$ & $(3,49)$ & $(0,41)$ & $(2,52)$ \\
\hline $\mathbf{R}^{2}$ & 0,81 & 0,81 & 0,46 & 0,44 & 0,92 & 0,91 & 0,70 & 0,62 & 0,43 & 0,52 & 0,09 & 0,12 \\
\hline
\end{tabular}

Anmerkungen: t-Werte in Klammern (White Heteroskedastie-robuste Standardfehler); *, ** und *** stehen für Signifikanz auf Zehn-, Fünf- bzw. Ein-Prozent-Niveau; Schätzungen enthalten als Kontrollvariablen die Arbeitslosenquote, das durchschnittliche Arbeitnehmerentgelt, die Schuldenhöhe pro Einwohner, sowie einen linearen Zeittrend. In der

Spezifikation mit dem Anteil der über 65-jährigen wird der Zeittrend sowie die Demographie-Variable mit einem Dummy für Niedersachsen interagiert. Aus den Schätzungen werden die wirtschaftlichen Strukturvariablen ausgeschlossen, wenn diese auf dem 10\%-Niveau insignifikant sind. Anzahl der Beobachtungen $=180(10 \mathrm{Länder} \times 18$ Jahre $)$. 


\section{E. Spezifikationstests}

Tabelle A3: Panel-Testverfahren

\begin{tabular}{|l|c|c|c|}
\hline Demografie-Variable & $\begin{array}{c}\text { F-Test auf } \\
\text { fixe Ländereffekte }\end{array}$ & $\begin{array}{c}\text { LM-Test auf zufällige } \\
\text { Ländereffekte }\end{array}$ & Hausman Test \\
\hline > 65 Jahre & $37,15 * * *$ & $132,29 * * *$ & $37,19 * * *$ \\
\hline$>$ 55 Jahre & $33,82 * * *$ & $131,60 * * *$ & $30,22 * * *$ \\
\hline
\end{tabular}

Anmerkungen: $* * *$ steht für Signifikanz auf Ein-Prozent-Niveau; Grundlage sind Schätzungen aus Tabelle A2.

Tabelle A4: Autokorrelation 1. Ordnung in Residuen der Panelschätzungen

\begin{tabular}{|l|c|c|c|}
\hline Demografie-Variable & Pooled OLS & Fixed Effects & Random Effects \\
\hline$>$ 65 Jahre & $\begin{array}{r}0,78 * * * \\
(11,12)\end{array}$ & $\begin{array}{r}0,68 * * * \\
(6,01)\end{array}$ & $\begin{array}{r}0,87 * * * \\
(11,77)\end{array}$ \\
\hline > 55 Jahre & $\begin{array}{r}0,78 * * * \\
(11,34)\end{array}$ & $\begin{array}{r}0,73 * * * \\
(6,93)\end{array}$ & $\begin{array}{r}0,83 * * * \\
(9,60)\end{array}$ \\
\hline
\end{tabular}

Anmerkungen: t-Werte in Klammern; *** steht für Signifikanz auf Ein-Prozent-Niveau; (White Heteroskedastie-robuste) Schätzungen mit Residuen aus Schätzungen in Tabelle A2 zur Ermittlung der Autokorrelationskoeffizienten für Cochrane-Orcutt-Schätzungen. Regressionen mit Konstante sowie den ersten beiden Verzögerung der Residuen (Autokorrelation 2. Ordnung in keinem Fall signifikant auf 10\%-Level). 